\title{
High power and high repetition rate pulse generation using self injection-locking in Fabry-Perot Laser diode
}

\author{
Xiaohui Fang, P. K. A. Wai, Chao Lu, H. Y. Tam, and Xinyong Dong \\ Photonics Research Center and Department of Electronic and Information Engineering \\ The Hong Kong Polytechnic University, Hung Hom, Kowloon, Hong Kong \\ enxfang@eie.polyu.edu.hk
}

\begin{abstract}
A $139.6 \mathrm{GHz}$ transform limited pulse train with peak power of $120 \mathrm{~mW}$ is generated using self injection-locking of a Fabry-Perot Laser diode. The pulsewidth is $1.6 \mathrm{ps}$ and the time-bandwidth product is 0.34 .

OCIS code: (140.3520) Laser, injection-locked, (190.7110) Ultrafast, nonlinear optics
\end{abstract}

\section{Introduction}

High bit-rate pulse trains have many important applications in ultrahigh-speed optical communication systems. Active mode locking [1] or modulation instability in erbium-doped fiber ring lasers (ED-FRL) [2-3] are attractive methods to generate high speed pulse trains, but the output power is not very high. High repetition pulse generation based on nonlinear propagation of a dual-frequency beat-signal within a long piece of dispersion decreasing fiber requires two DFB lasers [4]. In this paper, we report a simple method to generate high power and high repetition rate pulse train based on self injection-locking in a commercially available Fabry-Perot laser diode (FP-LD). Two-mode self injection-locking in a FP-LD is achieved by connecting a dc driven FP-LD externally with two uniform fiber Bragg gratings (FBGs). The two self injection-locked modes interfere and evolve into a high power high repetition rate pulse train through interplay between self-phase modulation and anomalous dispersion in a long piece of highly nonlinear fiber (HNLF). The repetition rate and peak power of the transformed-limited pulse train is 139.6 $\mathrm{GHz}$ and $120 \mathrm{~mW}$ respectively.

\section{Experiment}

Figure 1 shows the experimental setup for the proposed high repetition rate pulse generation based on a self injection-locking of a FP-LD. We used a commercially available FP-LD with center wavelength of 1550 $\mathrm{nm}$ and threshold current of $8 \mathrm{~mA}$. The dc bias current is chosen to be $10.4 \mathrm{~mA}$. The multimode output of the FP-LD is injected into two uniform FBGs through a 1:1 coupler. The Bragg wavelengths of the two FBGs are tuned to coincide with two different longitudinal modes of the FP-LD. The two FBGs therefore reflect the power of the two longitudinal modes back to FP-LD, thus forming a two-mode self injection-locked output. The self injection-locked output can be optimized by adjusting the polarization controllers PC1 and PC2.

The self injection-locked FP-LD output is obtained from Arm 1 of the coupler (see Fig. 1). The two self injection-locked modes have similar polarization and will therefore beat. The beating signal is amplified by an EDFA with maximum output power of $\sim 340 \mathrm{~mW}$, and then propagates through a $1146 \mathrm{~m}$ long HNLF. The dispersion coefficient of the HNLF is $2.6 \mathrm{ps} / \mathrm{nm} / \mathrm{km}$ at $\sim 1550 \mathrm{~nm}$ and the nonlinear coefficient is $\sim 14$ $\mathrm{W}^{-1} \mathrm{~km}^{-1}$. Through interplay between four wave mixing and group velocity dispersion (GVD) in the HNLF, a high average power, high repetition pulse train is formed at the end of HNLF. The $10 \%$ output signal from a 1:9 coupler at the end of the HNLF is sent to an optical spectrum analyzer (OSA) with resolution of $0.01 \mathrm{~nm}$. The $90 \%$ output power is sent to an auto-correlator for autocorrelation trace measurement.

\section{Results and discussion}

Figure 2(a) shows the free running spectrum of the FP-LD. The center wavelength is around $1546.5 \mathrm{~nm}$. Figure 2(b) shows output spectrum of the two adjacent simultaneously self injection-locked longitudinal modes at Arm 1. The wavelengths of the two modes are

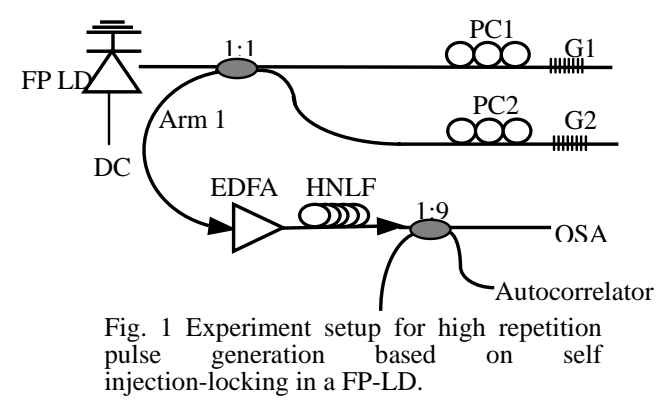




\section{CTuC1.pdf}

$1550.91 \mathrm{~nm}$ and $1552.03 \mathrm{~nm}$. The wavelength spacing is $1.12 \mathrm{~nm}$. Side mode suppression ratio (SMSR) is $\sim 38 \mathrm{~dB}$ and the output power is about $-26.39 \mathrm{dBm}$

The two self injection-locked longitudinal modes have the same polarization at Arm 1 because the FP-LD output is TE-polarized. The two longitudinal modes beat and experience both four wave mixing and GVD in the HNLF after amplification by the EDFA. Figure 3(a) shows the output spectrum from the $10 \%$ arm of the 1:9 coupler. From the spectrum, side bands are generated on both sides of the two original FP-LD modes as a result of FWM. The wavelength spacing between these side modes is $\sim 1.12 \mathrm{~nm}$. The $3 \mathrm{~dB}$ bandwidth of the spectrum is about $1.7 \mathrm{~nm}$. Figure 3(b) shows the corresponding autocorrelation trace of the pulse train. From Fig. 3(b), the peak-to-peak pulse spacing is 7.16 ps. This corresponds to a repetition rate of $139.6 \mathrm{~Gb} / \mathrm{s}$. The FWHM of the central pulse in the autocorrelation trace is $\sim 2.3 \mathrm{ps}$. Assuming a Gaussian profile, the FWHM of the corresponding individual pulse in the train is about 1.6 ps. This gives a time-bandwidth product of $\sim 0.34$ indicating a transform-limited pulse train. The average output power is $\sim 14.5 \mathrm{dBm}$. The peak power is therefore $\sim 120 \mathrm{~mW}$. The pulsewidth and time-bandwidth product can be varied by changing the pump power of EDFA. Figure 4 shows the pulsewidth (triangles) and time-bandwidth product (squares) versus the output power. From Fig. 4, the balance point of self-phase modulation and GVD is at output power of $\sim 14 \mathrm{dBm}$, where the pulse width is $\sim 1.6 \mathrm{ps}$ and time-bandwidth product is $\sim 0.34$.
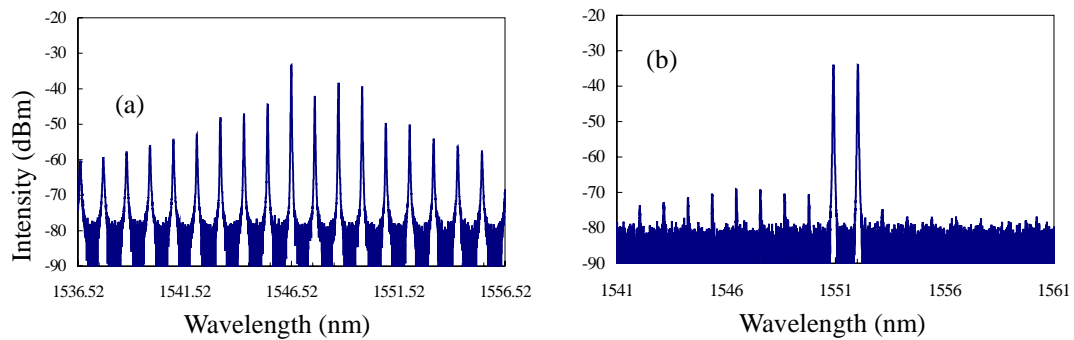

Fig. 2 (a) Free running spectrum of FP LD and (b) two simultaneously self injection-locked FP-LD modes at wavelengths of $1550.91 \mathrm{~nm}$ and $1552.03 \mathrm{~nm}$.
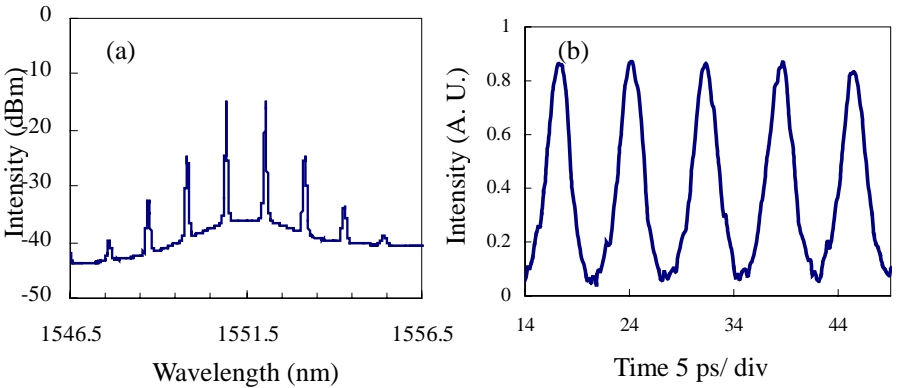

Fig. 3 (a) Output spectrum and (b) the corresponding autocorrelation trace aftel the HNLF.

\section{Conclusion}

We report a high output power, high repetition rate, and transform-limited optical pulse train generation based on simultaneously self injection-locking of two longitudinal modes of a FP-LD. The repetition rate is 139.6 GHz, the pulse width is $\sim 1.6 \mathrm{ps}$ and the time-bandwidth product is $\sim 0.34$.

\section{Acknowledgement}

This work is supported by The Hong Kong Polytechnic University (Project No. G-YX79).

\section{Reference}

1. N. Onodera, Supermode beat suppression in harmonically mode-locked erbium-doped fibre ring lasers with composite cavity structure, IEE Electron. Lett. 33, 962-963 (1997)

2. P. Francp, F. Fontana, I. Cristiani, M. Midrio and M. Romagnoli, self-induced modulational-instability laser Opt. Lett., 20, 2009-2011 (1995)

3. S. Zhang, F. Lu, X. Dong, P. Shum, X. Yang, X. Zhou, Y. Gong, and C. Lu, Passive mode locking at harmonics of the free spectral range of the intracavity filter in a fiber ring laser, Opt. Lett., 30, 2852-2854 (2005)

4. S. V. Chernikov, D. J. Richardson, R. I. Laming, E. M. Dianov and D. N. Payne, 70 Gbit/s Fiber based source of fundamental solitons at 1550 nm, Electron. Lett., 28, 1210-1211(1992). 